\title{
A Yeast STE11 Homologue CoMEKK1 Is Essential for Pathogenesis-Related Morphogenesis in Colletotrichum orbiculare
}

\author{
Ayumu Sakaguchi, Gento Tsuji, and Yasuyuki Kubo \\ Laboratory of Plant Pathology, Graduate School of Life and Environmental Sciences, Kyoto Prefectural University, \\ Kyoto 606-8522, Japan
}

Submitted 13 February 2010. Accepted 2 August 2010.

\begin{abstract}
Several signal transduction pathways, including mitogenactivated protein kinase (MAPK) pathways, are involved in appressorium development in Colletotrichum orbiculare, the causal agent of cucumber anthracnose disease. In this study, CoMEKK1, a yeast MAPK kinases (MAPKK) kinase STE11 homolog, was identified as a disrupted gene in an Agrobacterium tumefaciens-mediated transformation mutant. The phenotype of comekk1 disruptant was similar to that of cmk1, a Saccharomyces cerevisiae Fus3/Kss1 MAPK homolog mutant. Moreover, comekk1 and cmk1 mutants were sensitive to high osmotic and salinity stresses, indicating that Comekk1p/Cmk1p signal transduction is involved in stress tolerance. The transformants of the wild type and the comekk1 mutant expressing a constitutively active form of the CoMEKK1 showed slower hyphal growth and abnormal appressorium formation, whereas those of the cmk1 disruptant did not. A Cmk1p-green fluorescent protein (GFP) intracellular localization experiment indicated that nuclear localization of the Cmk1p-GFP fusion protein induced by salt stress was diminished in comekk1 mutants. These results indicate that Comekk1p functions upstream of Cmk1p.
\end{abstract}

Eukaryotic cells are constitutively stimulated by environmental signals such as growth factors, physical and chemical components, and nutrient starvation. The cells respond to these signals to adapt to specific environmental conditions. Plantpathogenic fungi possess signal transduction systems to respond to environmental cues. On recognition of the appropriate signals, the plant-pathogenic fungi develop pathogenesis-related structures via signal transduction pathways. Mitogen-activated protein kinase (MAPK) pathways are involved in transducing environmental cues and regulate cell differentiation in the plant-pathogenic fungi (Zhao et al. 2007). MAPK are usually activated by MAPK kinases (MAPKK or MEK), which are activated, in turn, by MEK kinases (MAPKKK or MEKK). These MAPK cascades (MEKK-MEK-MAPK) are conserved in eukaryotic cells and have been studied in several organisms. In the budding yeast Saccharomyces cerevisiae, MAPKKK STE11 initiates activation of the remainder of the MAPK cascade, MAPKK STE7, and MAPK FUS3, in response to pheromones (Chen and Thorner 2007). In plant-pathogenic

Correspondence author: Yasuyuki Kubo; E-mail: y_kubo@kpu.ac.jp

* The $\boldsymbol{e}$-Xtra logo stands for "electronic extra" and indicates that Figures $1,4,5,6,7$, and 8 appear in color online. fungi, this pheromone response-related MAPK cascade homolog is essential for pathogenicity (Zhao et al. 2007). In the rice blast fungus Magnaporthe oryzae, for instance, the MST11MSt7-PMK1 cascade is involved in appressorium formation and infective hyphae development in host leaves (Zhao et al. 2007). In Ustilago maydis, a causal agent of corn smut disease, the Ubc4/Kpp4-Fuz7/Ubc5-Ubc3/Kpp2 cascade is involved in formation of conjugation tubes, filamentous growth, and pathogenicity (Andrews et al. 2000; Banuett and Herskowitz 1994; Mayorga and Gold 1998, 1999; Müller et al. 1999, 2003).

Colletotrichum orbiculare is the causal agent of cucumber anthracnose disease. The infection process is initiated on recognition of an appropriate surface. This fungus undergoes a series of changes in morphology upon recognizing appropriate signals, including formation of a specialized infection structure, the appressorium. Metabolisms essential for appressorium function include melanin synthesis (Kubo and Furusawa 1991; Kubo et al. 1996; Perpetua et al. 1996; Takano et al. 1995; Tsuji et al. 2000), peroxisome metabolism (Asakura et al. 2009; Kimura et al. 2001; Fujihara et al. 2010), cellular polarity (Sakaguchi et al. 2008, 2010), and cell wall integrity (Tanaka et al. 2007, 2009).

Several signal-transduction-related genes associated with these morphological changes have been characterized in $C$. orbiculare and it has been shown that the MAPK and cyclic AMP (cAMP) signaling pathways are linked to infectionrelated morphological changes in this fungus. The budding yeast SLT2 homolog MAF1 is essential for appressorium development but not for pathogenicity (Kojima et al. 2002). A pheromone response MAPK gene FUS3/KSS1 homolog, CMK1, was also identified, and the $c m k l$ mutant displayed conidial germination, appressorium formation, and invasive growth (Takano et al. 2000). CST1, a homolog of transcriptional factor STE12, the downstream target of FUS3/KSS1, is essential for formation of infection pegs from the appressoria (Tsuji et al. 2003b). However, upstream MAPK factors have not been identified for C. orbiculare.

The cAMP signaling pathway also plays a pivotal role in transducing environmental cues for cell development. Moreover, crosstalk between protein kinase A (PKA) and MAPK signaling is a broad phenomenon in eukaryotes (Stork and Schmitt 2002). The cAMP signaling pathway plays a critical role in regulating conidial germination and pathogenicity in C. orbiculare (Takano et al. 2001; Yamauchi et al. 2004). Although mutants of the $R P K 1$ gene encoding the PKA regulatory subunit form normal melanized appressoria, they are defective in generating penetration hyphae (Takano et al. 2001). Mutants of adenylate cyclase and PKA genes have also been 
shown to be defective in conidial germination and pathogenicity (Yamauchi et al. 2004).

In this study, we identified a putative $C$. orbiculare MAPKK kinase encoding gene CoMEKK1, which shows high homology with $S$. cerevisiae STE11 based on mutant screening. By analyzing comekkl disrupted mutants, we elucidated that CoMEKK1 is essential for pathogenesis-related morphogenesis and, thus, pathogenesis.

\section{RESULTS}

\section{Identification of a STE11 homolog CoMEKKI in C. orbiculare.}

CoMEKK1 was identified by screening Agrobacterium tumefaciens-mediated transformation (AtMT) T-DNA mutants of C. orbiculare for defective appressorium development on cucumber leaves. We obtained a mutant responsible for defective appressorium development named KENH-2 (Fig. 1A). To isolate genomic DNA segments adjacent to a T-DNA of the KENH-2 strain, T-DNA insertion regions in mutant genomic DNA were amplified by thermal asymmetrical interlaced polymerase chain reaction (TAIL-PCR). Sequence analysis showed that the sequenced TAIL-PCR products had significant homology with STE11 in S. cerevisiae; they were designated as CoMEKK1. A primer pair was designed to isolate a CoMEKK1 genomic clone from a genomic cosmid library of C. orbiculare. The full-length sequence of CoMEKK1 was de-
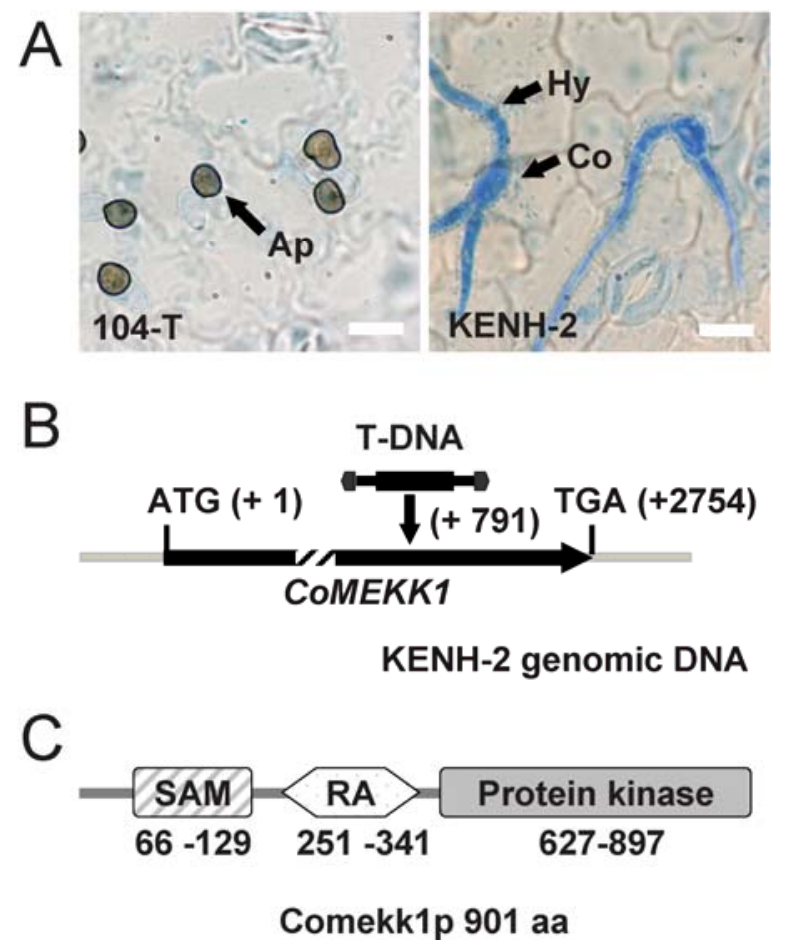

Fig. 1. Identification of the CoMEKK1 gene in Colletotrichum orbiculare. A, Morphological development of the KENH-2 strain on cucumber cotyledons. The wild-type $C$. orbiculare 104-T and the comekk1-disrupted mutant KENH-2 strains were assayed. Conidial suspensions were inoculated on the abaxial surfaces of cucumber cotyledons at $24^{\circ} \mathrm{C}$ for $24 \mathrm{~h}$. B, Schematic representation of the CoMEKK1 locus in the KENH-2 strain. Exons and an intron are indicated as closed boxes and a hatched box, respectively. The open reading frame of CoMEKK1, starting with the predicted initiator ATG (designated as +1 ) and ending with a TGA termination codon at $+1,668$, is indicated. The T-DNA insertion position is indicated at +791 . C, Schematic representation of Comekk1p. The lower values indicate the position of the sterile $\alpha$ motif (SAM) domain, Ras-association (RA) domain, and catalytic protein kinase domain. These domains were identified by searching the PROSITE database. termined. Based on the preferred translational initiation codon, CoMEKK1 was predicted to encode a protein of 901 amino acids (aa) (Fig. 1B). Like STE11 in S. cerevisiae, CoMEKK1 encodes a sterile $\alpha$ motif (SAM) domain at the $\mathrm{N}$ terminal (66 to 129 aa) and a C-terminal protein kinase domain (627 to 897 aa). Downstream of the SAM domain, CoMEKK1 also encodes a Ras-association (RA) domain (251 to 341 aa), which is conserved in STE11 homologs from other filamentous fungi (Fig. 1C) (Zhao et al. 2007).

To investigate distribution of CoMEKK1 homologs in other fungal genomes, we searched the National Center for Biotechnology Information nonredundant protein database using BLASTP. The deduced amino acid sequence of CoMEKK1 showed high similarity with amino acids of the STE11 homolog in $S$. cerevisiae and filamentous fungi such as M. oryzae, U. maydis, Aspergillus nidulans, and Neurospora crassa. A phylogenetic tree, based on the amino acid sequences of CoMEKK1 homologs and other MAPK kinases, indicated that Comekk1p was classified with the Ste11 MAPKK kinase family (Fig. 2).

\section{CoMEKK1 is essential}

for infection-related morphogenesis and plant infection.

To determine whether CoMEKK1 functioned in the Cmk1 MAPK pathway of $C$. orbiculare, we generated CoMEKKI gene-disrupted mutants. An Agrobacterium tumefaciens binary gene replacement vector (revS-MEKK1AH3) was introduced into the wild-type $C$. orbiculare 104-T by AtMT (Fig. 3A). Gene replacement and reintroduction of CoMEKK1 was confirmed by DNA gel blot analysis (Fig. 3B).

Phenotypes of the comekkl mutant were quite similar to those of the cmkl mutant. The mycelial growth rate of the comekkl mutant was also similar to that of the wild-type strain 104-T on potato dextrose agar (PDA) medium (Table 1). On the other hand, conidiation was reduced in the comekkl mutant compared with the wild-type strain (Table 1), and conidial morphology was quite similar to $c m k 1$, showing round form

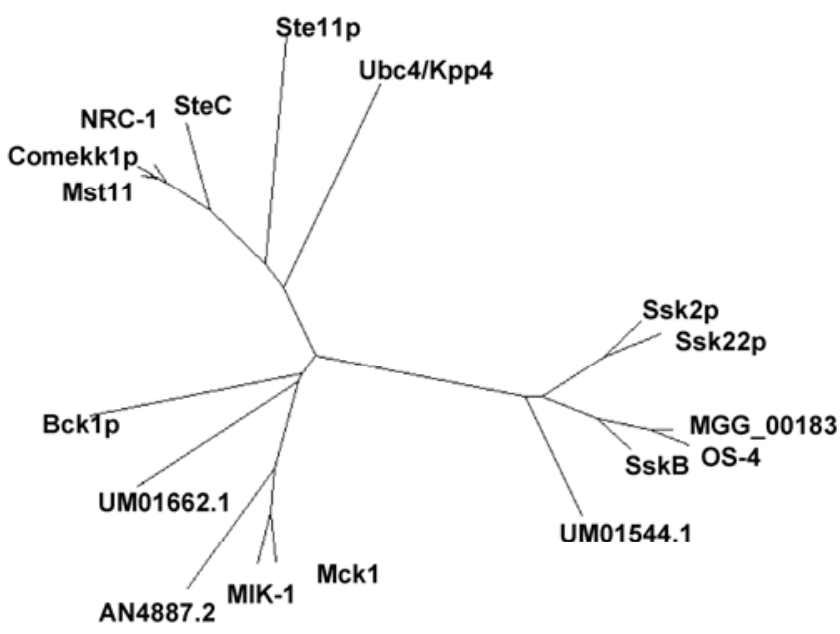

0.1

Fig. 2. Phylogenic analysis of mitogen-activated protein kinase kinase (MEK) kinases in yeast and filamentous fungi. The phylogenetic tree compiled from the amino acid sequences of the putative MAPKKK homologs among fungi was drawn using TreeView software. The phylogenetic analysis was performed using ClustalW based on sequences from Saccharomyces cerevisiae (Ste11p, Bck1p, Ssk2p, and Ssk22p), Magnaporthe oryzae (Mst11, Mck1, and MGG_00183), Neurospora crassa (NRC-1, MIK-1, and Os-4), Aspergillus nidulans (SteC, SskB, and AN4887.2) and Ustilago maydis (Ubc4/Kpp4, UM01662.1, and UM01544.1). The scale bar indicates the evolutionary distance. 
with shorter length of the major axis of conidial ellipse. To investigate infection-related morphogenesis of the comekkl mutant, we observed conidial germination and appressorium development on glass slides. In the wild type, ectopic transformants, and CoMEKK1 reintroduced transformants, approximately $90 \%$ of conidia germinated and formed darkly



Fig. 3. Gene disruption of CoMEKK1 in Colletotrichum orbiculare. A, CoMEKK1 gene disruption by homologous recombination with the revsMEKKAH3 vector in which the AH3 fragment (AMP::HPH cassette) was inserted into the CoMEKK1 gene. Through double crossover, an approximately 10-kb KpnI fragment containing wild-type CoMEKK1 was predicted to be replaced by an approximately $13-\mathrm{kb}$ fragment containing the AH3 fragment. B, Southern blot analysis of comekk1 disruption. Genomic DNA was digested with $K p n I$ and probed with an internal fragment of CoMEKK1. WT, wild-type 104-T strain; R1-2, the comekk1 mutant; E1, the CoMEKK1 ectopic transformant; $\mathrm{C} 1-2$, the CoMEKK1 t reintroduced transformant into the comekk1 mutant.

Fig. 4. Infection-related morphogenesis and pathogenicity assay of the comekk1 mutants. A, Microscopic observation of germlings of the comekk1 mutant. Conidial suspensions of each strain in distilled water or $0.1 \%$ yeast extract solution were incubated on multiwell glass slides at $24^{\circ} \mathrm{C}$ for $24 \mathrm{~h}$. 104-T, the wild-type strain; RMKK-1, the comekk1 mutant; Ap, appressoria; Co, conidia; Hy, hyphae. Scale bars $=10 \mu \mathrm{m}$. B, Percentage of conidial germination and appressorium formation by the comekkl mutants. Conidial suspensions of each strain in distilled water or $0.1 \%$ yeast extract solution were incubated on multiwell glass slides at $24^{\circ} \mathrm{C}$ for $24 \mathrm{~h}$. Approximately 200 conidia were observed per well; three independent experiments were performed and mean and standard deviations were noted. 104-T, the wild-type strain; RMKK-1, 2, the comekk1 mutants; EMKK-1, the CoMEKK1 ectopic transformant; CMKK-1, 2; the CoMEKK1 reintroduced transformants; $c m k l \Delta$, the $c m k 1$ mutant. C, Pathogenicity assay of comekk1 mutants on wounded leaves. Mycelial blocks were placed on wound sites prepared by scratching the leaves with a sterile toothpick and inoculated at $24^{\circ} \mathrm{C}$ for 6 days. 104-T, the wild-type strain; RMKK-1, 2, the comekk1 mutants; EMKK-1, the CoMEKK1 ectopic transformant; CMKK-1, 2; the CoMEKK1 reintroduced transformants; cmk1 $\Delta$, the $c m k l$ mutant. melanized appressoria within $24 \mathrm{~h}$ (Fig. 4A). In contrast, the germination frequency of the conidia in the comekkl mutant was approximately $1 \%$ after $24 \mathrm{~h}$ of incubation. This result was similar to that of the cmkl mutant (Takano et al. 2000). Conidia of the $\mathrm{cmkl}$ mutant germinate in yeast extract solution without forming appressoria, indicating that the Cmk1 MAPK pathway was not crucial for nutrient signal response (Takano et al. 2000). Therefore, to assess whether Comekk1p is involved in nutrient signal responses, conidia of the comekkl mutant were incubated in yeast extract solution. The conidia of

the comekkl mutants germinated with comparable frequency to the wild-type strain in yeast extract solution without forming appressoria like the cmklmutant (Fig. 4A and B).

$C M K 1$ is required for invasive growth in host cucumber leaves (Takano et al. 2000). To assess whether CoMEKKI is required for invasive growth in host leaves, comekkl mutants were inoculated onto wounded cucumber leaves. Mycelial blocks were incubated at the wound site, which was induced by scratching with a toothpick. The wild-type ectopic transformants and CoMEKK1-reintroduced transformants formed lesions at 6 days, whereas the comekkl and cmkl mutants did not (Fig. 4C).

The Ste11p function in the high osmolarity glycerol (HOG) pathway in S. cerevisiae (Posas and Saito 1997) and the Pmk1 MAPK pathway in $M$. oryzae regulates high osmolarity responses independent of the Osm1 MAPK pathway during vegetative growth (Zhao et al 2005). However, it is unclear whether the Cmk1 MAPK pathway is involved in the osmotic stress response of $C$. orbiculare. In this study, the growth rate of the comekkl and cmkl mutants was lower than that of the wildtype strain on PDA medium containing $1 \mathrm{M}$ sorbitol as a high osmotic stressor (Fig. 5). Furthermore, the comekkl and cmkl mutants showed lower tolerance to salt stress. On PDA medium containing $0.3 \mathrm{M} \mathrm{NaCl}$, the growth rate of the comekkl and cmkl mutants was lower than that of the wild type (Fig. 5).

\section{A constitutively active CoMEKK1 allele showed reduction of vegetative growth and abnormal appressorium formation that is dependent on $C M K 1$.}

To investigate whether Comekk1p activates Cmk1p in $C$. orbiculare, a putative constitutively active allele of CoMEKK1 was constructed. We anticipated that constitutively active Comekk1p would affect some cellular responses in the wildtype or comekkl mutants if Comekk1p acts upstream of Cmk1p, and that these responses would not be expressed by the cmkl mutant. Therefore, Comekk1p hyperactive strains were generated from the wild-type strain and the comekkl and cmk1 mutants. In the STE11-4 allele of S. cerevisiae, a constitutively active allele of STE11, threonine 596, was replaced by isoleucine adjacent to the invariant Asp-Phe-Gly (DFG) triplet in the subdomain, VII kinase domain (Stevenson et al. 1992). Based on the STE11-4 data, we replaced serine 775 with isoleucine adjacent to the DFG triplet in the kinase domain of Comekk1p and named this encoding gene CoMEKK1-4.

The CoMEKK1-4-introduced transformants, WME4 and mME4, obtained from the wild-type and comekkl mutants,

Table 1. Growth rate and conidiation of Cmk1 MAPK kinase signaling mutants in Colletotrichum orbiculare

\begin{tabular}{|c|c|c|}
\hline Strain $^{a}$ & $\begin{array}{c}\text { Vegetative growth } \\
(\mathrm{mm} / \mathbf{3} \text { days })^{\mathbf{b}}\end{array}$ & $\begin{array}{c}\text { Conidia } \\
\left(\times 10^{6} / \text { colony }\right)^{c}\end{array}$ \\
\hline $104-\mathrm{T}$ & $7.73 \pm 0.27$ & $18.9 \pm 6.04$ \\
\hline RMKK-1 & $7.73 \pm 0.10$ & $0.18 \pm 0.06$ \\
\hline RMKK -2 & $7.73 \pm 0.03$ & $0.16 \pm 0.07$ \\
\hline EMKK -1 & $7.93 \pm 0.07$ & $23.8 \pm 4.52$ \\
\hline CMKK -1 & $7.83 \pm 0.19$ & $18.4 \pm 4.59$ \\
\hline CMKK -2 & $7.63 \pm 0.18$ & $16.7 \pm 5.43$ \\
\hline cmkl $\Delta$ & $7.78 \pm 0.16$ & $0.19 \pm 0.13$ \\
\hline
\end{tabular}

a Strains: 104-T, the wild-type strain; RMKK-1 and 2, comekk1 mutants; EMKK-1, CoMEKK1 ectopic transformant; CMKK-1 and 2; CoMEKK1 reintroduced transformants; $c m k 1 \Delta, c m k 1$ mutant.

${ }^{\mathrm{b}}$ Each strain was grown on potato dextrose agar at $24^{\circ} \mathrm{C}$. Strains were measured from colony edge incubated for 3 days to colony edge incubated for 6 days. Five colonies in each strain were measured and the mean and standard deviation were calculated.

${ }^{\mathrm{c}}$ Production of conidia was measured in 7-day-old culture. Conidia of five colonies in each strain were counted and the mean and standard deviation were calculated. respectively, showed reduced hyphal growth on PDA. The hyphal growth of WME4 and mME4 was slower than that of the parent strains. Moreover, WME4 and mME4 showed increased tolerance to salt and osmotic stresses compared with the wild-type strain (Fig. 6A). On the other hand, hyphal growth and stress responses in the CoMEKK1-4 introduced cmkl mutant (cME4) showed no distinct difference in these characteristics compared with the cmkl mutants (Fig. 6A). Additionally, we investigated whether constitutively active Comekk1p affects infection-related morphogenesis (Fig. 6B and $\mathrm{C}$ ). The conidia of WME4 and mME4 germinated and formed appressoria, and 20 to $40 \%$ of the appressoria of these strains displayed a morphologically aberrant phenotype with lateral germination from the appressoria. In contrast, cME4 was not affected by hyperactive Comekk1p and conidial germination frequency was very low. These results were similar to those for the cmkl mutant (Fig. 6B and C).

A
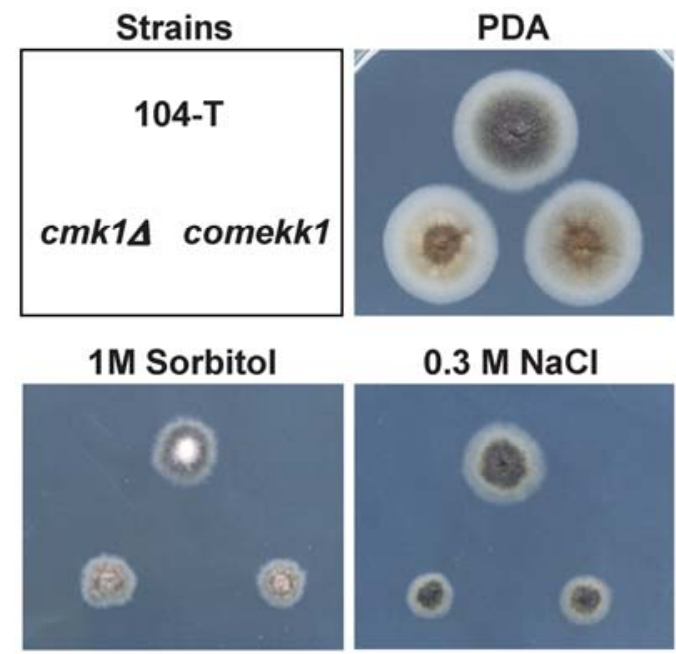

$0.3 \mathrm{M} \mathrm{NaCl}$



B

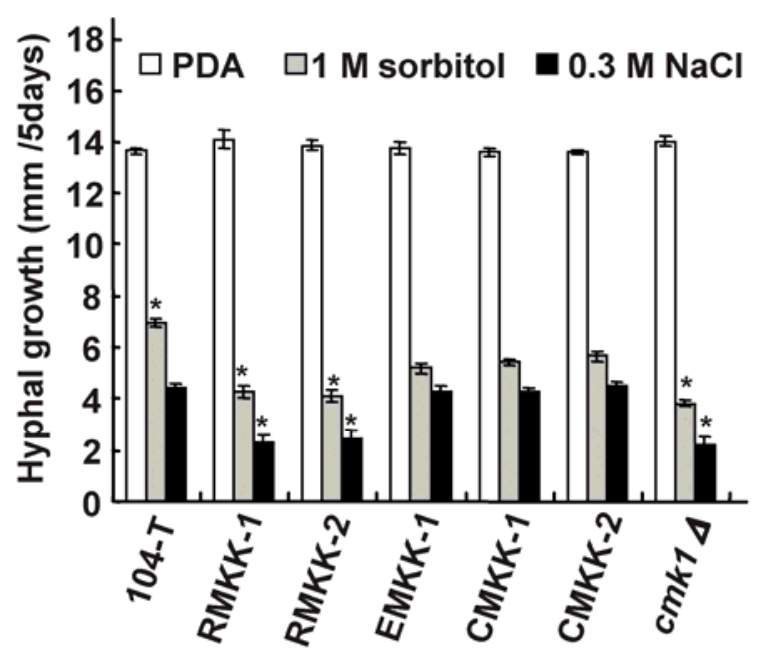

Fig. 5. Sensitivity of the Cmk1 mitogen-activated protein kinase (MAPK) signaling mutant to salt or osmotic stresses. A, Growth of the Cmk1 MAPK signaling mutants on the stress medium. Each strain was grown on potato dextrose agar (PDA) containing $1 \mathrm{M}$ sorbitol or $0.3 \mathrm{M} \mathrm{NaCl}$ at $24^{\circ} \mathrm{C}$ for 5 days. $104-\mathrm{T}$, the wild-type strain; RMKK-1, the comekk1 mutants; cmk1 1 , the $c m k 1$ mutant. B, Growth rate of each strain on stress medium. Colony extension from 2 to 7 days after inoculation was measured. Five colonies from each strain were measured and the mean and standard deviations were calculated. 104-T, the wild-type strain; RMKK1, 2, the comekk1 mutants; EMKK-1, the CoMEKK1 ectopic transformant; CMKK-1, 2; the CoMEKK1 reintroduced transformants; cmk1 1 , the $c m k 1$ mutants. Asterisks represent significant differences between the ectopic control and each strain. Student's $t$ test: * indicates $P<0.001$. 


\section{Nuclear localization of Cmk1-GFP is affected} by Comekk1p.

Once activated by environmental cues, MAPK localize to nuclei and activate downstream targets. In $C$. orbiculare, Osc1 MAPK is transferred to the nuclei following stimulation by osmotic stress. The Fus3 MAPK localizes in the nuclei and cytoplasm. However, Fus3p is more abundant in the nuclei than in the cytoplasm when stimulated by mating pheromones (Blackwell et al. 2003). A CMK1-green fluorescent protein $(G F P)$ fusion gene was constructed to investigate whether signal transduction via Comekk1p controls localization of Cmk1p. The CMK1-GFP fusion gene, expressed under its native $C M K 1$ promoter, was introduced into the $c m k 1$ mutant. Using DNA blot analysis, we confirmed that the resulting transformant (CG1) contained one copy of the fusion gene (data not shown). The $c m k l$ mutant restored conidial germination, appressorium development, pathogenicity, and $\mathrm{NaCl}$ tolerance following the introduction of $C M K 1-G F P$, indicating that $C M K 1-G F P$ was functional (Fig. 7). When induction did not occur, CMK1-GFP was not localized at cellular specific sites in the hyphae and conidia of CG1, whereas it was effectively localized in the nuclei under conditions of $5 \mathrm{M} \mathrm{NaCl}$ salt stress (Fig. 8A). Next, we investigated whether localization of CMK1-GFP was affected in the comekk1 mutant or in the CG1 strain expressing CoMEKK1-4. The CMK1-GFP fusion gene was introduced into the comekk1 mutant RMKK1-1 strain (designated as the mCG1 strain) and CoMEKK1-4 was introduced into the CG1 strain (designated as the ACG1 strain). In the conidia of CG1 or ACG1 in $5 \mathrm{M} \mathrm{NaCl}$, GFP signals were conspicuous in the nuclei (Fig. 8B). The percentage of GFP signals observed in the nuclei was approximately 75 and $90 \%$ in the conidia of CG1 and ACG1, respectively (Fig. 8C). In contrast, localization of Cmk1-GFP in the nuclei in mCG1 was much lower than that of CG1 and ACG1; the percentage of GFP signals observed in the nuclei was approximately $35 \%$ (Fig. 8).

\section{DISCUSSION}

There has been an accumulation of data pertaining to MAPK signaling and pathogenicity in plant-pathogenic fungi (Xu 2000; $\mathrm{Xu}$ et al. 2007). MAPK signaling pathways are conserved in the plant-pathogenic fungi and yeasts. However, upstream components of the MAPK cascade in some plant
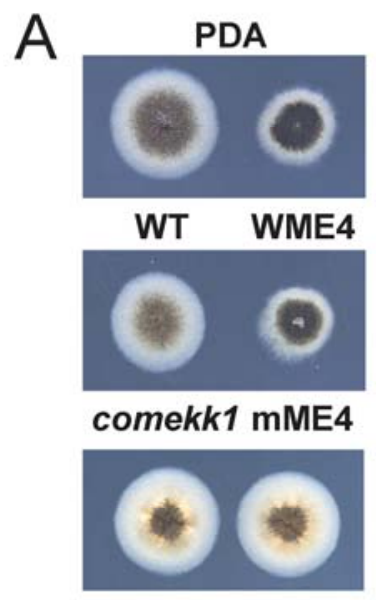

cmk14 cME4
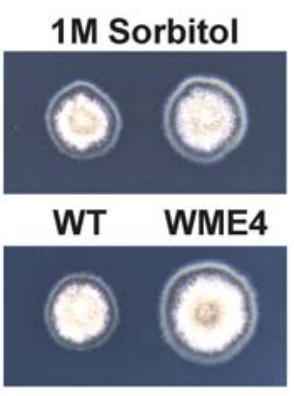

WT

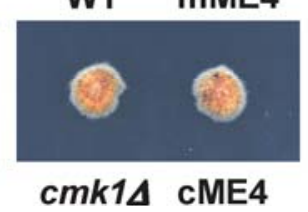

B



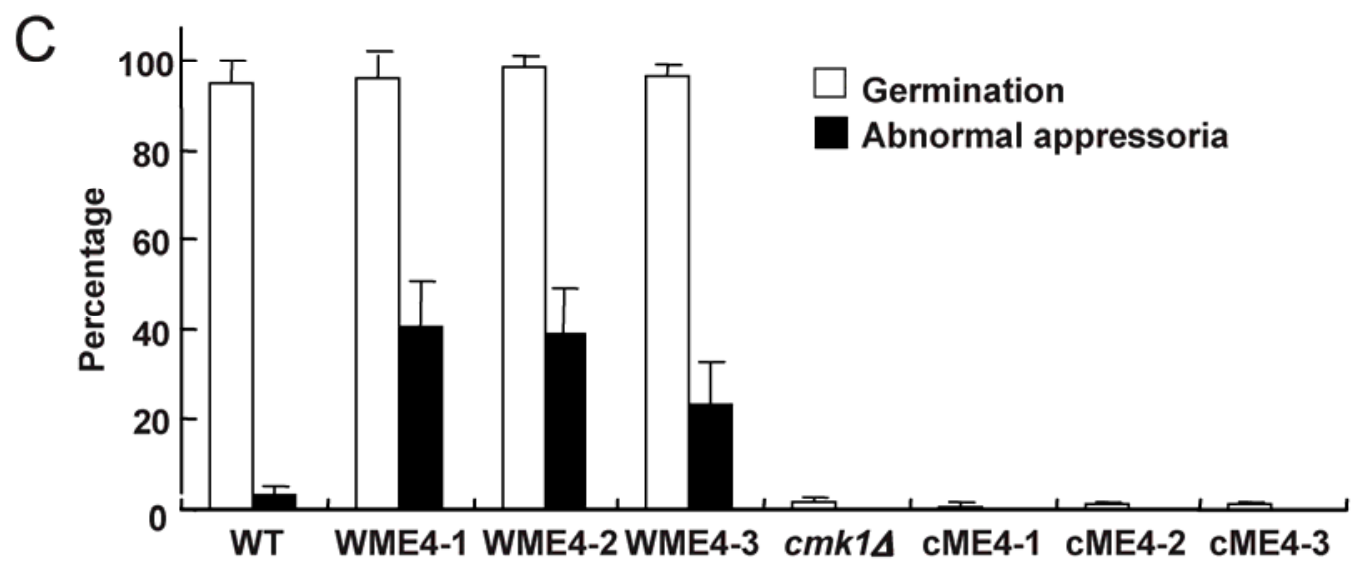

Fig. 6. Expression of the CoMEKK1-4 allele in mutants. A, Growth of the CoMEKK1-4 transformants on the stress medium. Each strain was grown on potato dextrose agar (PDA) containing $1 \mathrm{M}$ sorbitol at $24^{\circ} \mathrm{C}$ for 5 days. WT, the wild-type strain; comekk1, the comekk1 mutants; $\mathrm{cmk} 1 \Delta$, the $\mathrm{cmk} 1 \mathrm{mutants}$; WME4, CoMEKK1-4 introduced the wild type; mME4, CoMEKK1-4 introduced the comekk1 mutant; cME4, CoMEKK1-4 introduced the cmk1 mutant. B, Appressorium formation by the CoMEKK1-4 introduced strain. Ap, appressoria; Co, conidia. Scale bars = $10 \mu \mathrm{m}$. WT, the wild-type strain; ME4, CoMEKK1-4 introduced wild-type; mME4, CoMEKK1-4 introduced comekk1 mutant; cME4, CoMEKK1-4 introduced cmk1 mutant. Conidial suspensions of each strain prepared in distilled water were incubated on multiwell glass slides at $24^{\circ} \mathrm{C}$ for $24 \mathrm{~h}$. Ap, Appressoria; Co, Conidia. Scale bars $=10 \mu \mathrm{m}$. C, Percent conidial germination and abnormal appressorium formation. WT, the wild-type strain; ME4-1,2,3, CoMEKK1-4 introduced into the wild-type strain; cME4-1,2,3, CoMEKK1-4 introduced into the cmk1 mutant. Approximately 200 conidia were observed per well and three replicates were examined. Mean and standard deviations were calculated from three independent experiments. 
pathogens do not appear to be consistent with those of $S$. cerevisiae. In $S$. cerevisiae, PAK kinase Ste20p is required for Ste $11 \mathrm{p}$ activation in the mating and filamentous growth pathways (Chen and Thorner 2007). On the other hand, CHMI and MST20, the PAK kinase genes in M. oryzae, have been shown to be dispensable for appressorium formation and may not be involved in activating the Mst11-Mst7-Pmk1 MAPK pathway (Li et al. 2004). Thus, parts of the signaling pathways of the plant-pathogenic fungi, from signal recognition up to cellular response, are not consistent with $S$. cerevisiae.

In $C$. orbiculare, although two MAPK are involved in pathogenicity (Kojima et al. 2002; Takano et al. 2000), the upstream factors influencing these MAPK have not been identified. CoMEKK1 encodes the SAM domain at the $\mathrm{N}$ terminal, followed by the RA domain and protein kinase domain at the $\mathrm{C}$ terminal, similar to that for M. oryzae MST11, U. maydis $U b c 4 / K p p 4$, and other STE11 homologs (Andrews et al. 2000; Grimshaw et al. 2004; Muller et al. 2003; Zhao et al. 2005) and it has significant sequence similarity to those genes, suggesting that CoMEKK1 encodes MAPKK kinase. The phenotype of the comekkl disruption mutants showed reduced conidiation, inability to germinate and form appressoria, and
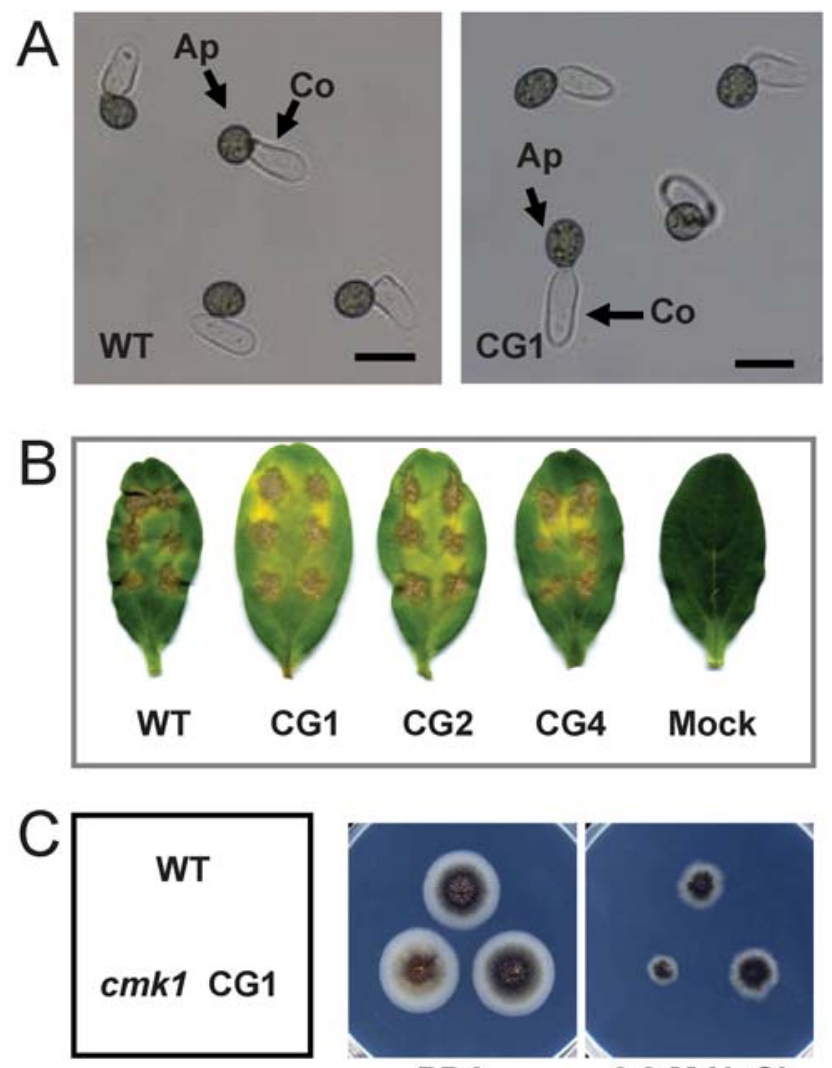

PDA

$0.3 \mathrm{M} \mathrm{NaCl}$

Fig. 7. Complementation test for pathogenicity and stress tolerance of cmkl mutant by introduction of the CMK1-green fluorescent protein $(G F P)$ fusion gene. A, Appressorium formation by the CMK1-GFP gene introduced transformant into the CG1 strain and the wild-type strain 104T. Conidial suspensions of each strain, prepared in distilled water, were incubated on multiwell glass slides at $24^{\circ} \mathrm{C}$ for $24 \mathrm{~h}$. Ap, Appressoria; $\mathrm{Co}$, Conidia. Scale bars $=10 \mu \mathrm{m}$. B, Pathogenicity assay of CMK1-GFP-introduced transformants on intact cucumber cotyledons. Conidial suspensions were droplet inoculated onto detached cucumber cotyledons and incubated at $24^{\circ} \mathrm{C}$ for 6 days. WT, wild-type 104-T; CG1-3, CMK1-GFP-introduced transformant into the $c m k 1$ mutant. Mock, distilled water control. C, Growth of CMK1-GFP-introduced transformants on the stress medium. Wild-type (WT), CMK1-GFP-introduced transformants (CG1), and cmkl mutants were grown on potato dextrose agar (PDA) containing $0.3 \mathrm{M}$ $\mathrm{NaCl}$ at $24^{\circ} \mathrm{C}$ for 5 days. defective invasive growth on host cucumber leaves. In the budding yeast, Ste11 MAPKK kinase, Ste7 MAPK kinase, and Fus3/Kss1 MAPK constitute a cascade and regulate pheromone response and the filamentous growth pathway (Chen and Thorner 2007). Phenotypes of the disruptant of $C M K 1$, the FUS3/KSS1 homolog of C. orbiculare, were similar to those of the comekkl mutant. Moreover, we showed that both the comekkl and cmkl mutants, were sensitive to high osmotic and salinity stresses. These data indicate that CoMEKK1 and $C M K 1$ regulate the same pathway involving infection-related morphogenesis, pathogenicity, and stress tolerance, and that there is a possibility that their encoded proteins are part of the same cascade.

We induced mutagenesis of CoMEKKI in vitro in the region adjacent to the invariant DFG triplet in the subdomain VII kinase domain, as detailed in the study by Stevenson and associates (1992). This kinase domain mutation close to the ATPbinding cleft was likely to escape inhibition by the autoinhibitory domain (van Drogen et al. 2000). Expression of the hyperactive CoMEKK1-4 allele affected several phenotypes of the wild-type strain and the comekkl mutant. Thus, it is suggested that repression of kinase activity by the autoinhibitory domain is conserved between Ste11p and Comekk1p.

Expression of the CoMEKK1-4 allele rescued the defect in conidial germination, appressorium formation, and osmotic stress tolerance in comekkl mutants but not in cmkl mutants. This result indicates that MEKK encoded by CoMEKK1 likely functions upstream of CMK1.S. cerevisiae Ste11p is involved in regulation of the Fus3/Kss MAPK pathway and the Hog1 MAPK pathway (Chen and Thorner 2007; Posas and Saito 1997). In C. orbiculare, the Osc1 MAPK pathway (a Hog1related pathway) regulates osmotic stress response (Kojima et al. 2004). Moreover, it has been shown in this study that the Cmk1 MAPK pathway also regulates osmotic tolerance. Introduction of CoMEKK1-4 into the wild-type $C$. orbiculare strain or the comekkl mutant conferred tolerance against osmotic stress while it did not have that effect on the cmkl mutant. These results support the hypothesis that Comekk1p is a direct upstream component of the Cmk1 MAPK pathway. The strains in which CoMEKK1-4 had been introduced also showed significant reduction in vegetative growth on PDA, indicating that proper regulation of the Cmk1 MAPK pathway is required for normal vegetative growth in this fungus. On the other hand, expression of MST7 $7^{\mathrm{S} 212 \mathrm{D}} \mathrm{T} 216 \mathrm{E}$, a dominant active MEK allele, did not affect vegetative growth in $M$. oryzae (Zhao et al. 2005). The differences in phenotype of the dominant active introduced strains suggest that MST7 and CoMEKK1 regulate different signaling pathways.

In this experiment, Comekk1p hyperactivation influenced appressorium morphogenesis. Previous reports have shown that phosphorylation of Mps1 MAPK is repressed by expression of the dominant active MST7 allele and by activation of Pmk1 during appressorium formation, suggesting that Pmk1 activation may have a negative effect on Mps1 phosphorylation in $M$. oryzae. Maf1, the Mps1 MAPK of $C$. orbiculare, regulates appressorium formation, and disruption of $M A F 1$ has been shown to cause defective appressorium development (Kojima et al. 2002). Therefore, there is the possibility that hyperactivation of Comekk1p negatively regulates phosphorylation of Maf1p and affects appressorium morphogenesis.

MAPK responses are dependent on activation by MEK and their subsequent translocation to the nuclei where they phosphorylate transcription factors. Signal-induced nuclear translocation has been observed in some MAPK (Chen and Thorner 2007). The budding yeast Hog 1 MAPK exhibits a dramatic translocation from the cytoplasm to the nuclei when the cells are subjected to hyperosmotic shock (Reiser et al. 1999). Ac- 
cordingly, OSC1 MAPK, the Hog1-related MAPK in $C$. orbiculare, has been shown to translocate into the nuclei after stimulation by the addition of fludioxonil or $\mathrm{KCl}$ (Kojima et al. 2004). On the other hand, several MAPK constitutively shuttle between the cytoplasm and nuclei and do not show an obvious nuclear concentration when activated. Fus3p constitutively shuttles between the cytoplasm and nuclei and is easily detectable in both compartments. A quantitative assay has shown increased nuclear localization of Fus3p in response to pheromone stimulation (Blackwell et al. 2003). In M. oryzae, although a slight GFP-Pmk1 signal was detected in the conidia, germ tubes, and vegetative hyphae, the signal increased in the nuclei of the appressoria where nuclear localization was observed (Bruno et al. 2004). On the other hand, strong Cmk1GFP signals were detected in the cytoplasm of vegetative hyphae and the conidia of $C$. orbiculare. The GFP signals were also detected in the nuclei of the conidia under conditions of high-salt stress. To test the possibility that localization of Cmk1-GFP was affected by Comekk1p activation, we examined Cmk1-GFP nuclear translocation in the comekkl mutant. Nuclear localization of Cmk1-GFP in the comekkl mutant was reduced compared with the wild-type or hyperactive Comekk1p strains, indicating that nuclear translocation of Cmk1 is dependent on Comekk1p activity.


$\square$ nuclei < cytoplasm



$\square$ nuclei < cytoplasm

Fig. 8. Involvement of CoMEKK1 in the localization of Cmk1-green fluorescent protein (GFP) in stress condition. A, Cmk1-GFP localized to nuclei in the CG1 strain. The conidia of the CG1 strain were observed in $5 \mathrm{M} \mathrm{NaCl}$ solution and stained with Hoechst $33342(5 \mathrm{mg} / \mathrm{ml})$ for $5 \mathrm{~min}$. Scale bars $=10 \mu \mathrm{m}$. B, Cmk1-GFP localized to nuclei in the ACG1 strain but not in the mCG1 strain. The conidia of each strain were observed in $5 \mathrm{M} \mathrm{NaCl}$ solution. Scale bars = $10 \mu \mathrm{m}$. C, Percent nuclear localization of Cmk1-GFP in conidia in distilled water (DW, upper graph) or $5 \mathrm{M} \mathrm{NaCl}$ solution (lower graph). More than 100 conidia harvested as suspension in $\mathrm{DW}$ or $\mathrm{NaCl}$ solution were observed per experiment. Four independent experiments were conducted and mean and standard deviations were noted. Cmk1 localization was categorized as follows: nuclei < cytoplasm, nuclei $=$ cytoplasm, and nuclei $>$ cytoplasm. GFP, strain expressing GFP; CG1, the CMK1-GFP-introduced strain into the wild-type. mCG1, CG1, the CMK1-GFP-introduced strain into the comekk1 mutant; ACG1, the CoMEKK1-4 introduced strain to CG1. 
In $S$. cerevisiae, the Ste11p is also involved in the HOG pathway (Posas and Saito 1997). CoMEKK1 gene mutation caused higher sensitivity against high osmotic stress, indicating that Comekk1p also regulates osmotic regulatory pathways in $C$. orbiculare. In our experiment, hyperactivation of Comekk1p conferred high osmotic tolerance to the wild type and the comekkl mutant but not to the cmkl mutant. Furthermore, the $c m k l$ mutant also showed increased sensitivity against high osmotic stress at level similar to that of comekk1. These results suggested that Comekk1p is required for Cmk1p function to respond to osmotic stress, and this regulation would be independent of the Osc1 MAPK pathway in C. orbiculare. However, to verify this, a phosphorylation assay of the MAPK kinase upstream of the Osc1 MAPK-like Ste11p would be essential.

In conclusion, our results suggest that Comekk1p MAPKK kinase acts upstream of Cmk1 MAPK and regulates stress tolerance, appressorium development, and pathogenesis in $C$. orbiculare.

\section{MATERIALS AND METHODS}

\section{Fungal and bacterial strains.}

Strain 104-T (MAFF240422) of C. orbiculare (Berk. \& Mont.) Arx (syn. C. lagenarium (Pass.) Ellis \& Halst.) was used as the wild-type strain. All $C$. orbiculare strains used in this study are listed in Table 2 and were cultured on PDA media (3.9\% [wt/vol] PDA; Difco Laboratories, Detroit) at $24^{\circ} \mathrm{C}$. Escherichia coli DH5- $\alpha$ was used as a host for gene manipulation. E. coli was cultured in Luria-Bertani media (Sambrook et al. 1989 ) at $37^{\circ} \mathrm{C}$. The medium was supplemented when necessary with ampicillin at $50 \mu \mathrm{g} / \mathrm{ml}$ or with kanamycin at $50 \mu \mathrm{g} / \mathrm{ml}$. A. tumefaciens $\mathrm{C} 58 \mathrm{C} 1$ was used to transform $C$. orbiculare by AtMT. A. tumefaciens was cultured in AB-minimal medium at $28^{\circ} \mathrm{C}$. The medium was supplemented when necessary with kanamycin at $50 \mu \mathrm{g} / \mathrm{ml}$ and rifampicin at $100 \mu \mathrm{g} / \mathrm{ml}$.

\section{Fungal transformation.}

The AtMT protocol was applied with slight modifications of the previously described method (Tsuji et al. 2003a). The hygromycin-resistant transformants were selected on PDA medium containing hygromycin $\mathrm{B}$ at $100 \mu \mathrm{g} / \mathrm{ml}$ (Wako Chemicals, Osaka, Japan), cefotaxim at $100 \mu \mathrm{g} / \mathrm{ml}$ (Wako Chemicals), and spectinomycin at $100 \mu \mathrm{g} / \mathrm{ml}$ (Wako Chemicals). The bialaphos-resistant transformants were selected on SD medium containing bialaphos at $10 \mu \mathrm{g} / \mathrm{ml}$ (Meiji Seika Kaisha, Ltd., Tokyo), cefotaxim at $100 \mu \mathrm{g} / \mathrm{ml}$, and spectinomycin at $100 \mu \mathrm{g} / \mathrm{ml}$. The sulfonylurea-resistant transformants were selected on SD medium containing chlorimuronethyl at $4 \mu \mathrm{g} / \mathrm{ml}$
(Chem Service, West Chester, PA, U.S.A.), cefotaxim at 100 $\mu \mathrm{g} / \mathrm{ml}$, and spectinomycin at $100 \mu \mathrm{g} / \mathrm{ml}$.

\section{Genomic DNA blot analysis.}

Total DNA of $C$. orbiculare was isolated from mycelia incubated in potato-sucrose liquid medium for 3 to 4 days, and DNA blot analysis was performed according to the method previously described (Takano et al. 1997). DNA digestion, gel electrophoresis, labeling of probes, and hybridization were performed according to the manufacturer's instructions and standard methods (Sambrook et al. 1989). DNA probes were labeled with digoxigenin (DIG)-dUTP using a BcaBEST DIG labeling kit (Takara Bio, Ohtsu, Japan). Hybridized DNA was detected with antiDIG antibody Fab fragments conjugated to alkaline phosphatase (Roche Diagnostics, Tokyo) and light emission from the enzymatic dephosphorylation of CDP-Star Detection Reagent (GE Healthcare, Tokyo) was detected using the LAS-1000 Plus Gel Documentation System (Fujifilm, Tokyo).

\section{Cloning and sequencing.}

The analysis of fungal genomic DNA flanking the T-DNA inserts was achieved using the TAIL-PCR protocol performed as described previously (Tsuji et al. 2003a). Amplified PCR products were analyzed by agarose gel electrophoresis and sequenced with the Big-Dye terminator cycle sequencing ready reaction kit (Applied Biosystems Japan Ltd., Tokyo) and an ABI PRISM 310 automated DNA sequencer (Applied Biosystems Japan Ltd.). To identify the T-DNA insertion gene in the KENH-2 strain, a TAIL-PCR protocol was applied to the mutant genomic DNA (Liu et al. 1995). Fungal genomic DNA flanking the T-DNA insert was analyzed as previously described (Tsuji et al. 2003a). Amplified PCR products were sequenced with the Big-Dye terminator cycle sequencing ready reaction kit and an ABI PRISM 310 automated DNA sequencer. Genomic clones of CoMEKK1 were selected from a cosmid library of $C$. orbiculare $104-\mathrm{T}$ by PCR using primer pair KEH2-S1 (5'-CATGCGAAGAGGTCATGAGA-3') and KEH2-AS1 (5'-ATCAAACGGTTACGCTCAGG-3') designed from the obtained DNA sequence. The sequencing of the entire region of the CoMEKK1 open reading frame was as follows. The EZ::TN <KAN-2> Insertion Kit (Epicenter Technologies, Madison, WI, U.S.A.) was used to insert the transposon containing the selection marker into the target DNA. The clones of the target DNA containing the $<\mathrm{KAN}-2>$ transposon were then sequenced with the Big-Dye terminator cycle sequencing ready reaction kit using primers KAN2-RP2 and KAN2-FP3. The CoMEKK1 sequence is available in the DDBJ database under accession no. AB546841.

Table 2. Fungal strains used in this study

\begin{tabular}{|c|c|c|}
\hline Strain & Description & Reference \\
\hline $104-\mathrm{T}$ & Wild-type strain of Colletotrichum orbiculare & NIAS, Japan Genebank MAFF240422 \\
\hline RMKK-1 & comekk1 disruptant of 104-T & This study \\
\hline RMKK-2 & comekk1 disruptant of 104-T & This study \\
\hline EMKK-1 & CoMEKK1 ectopic transformant of $104-\mathrm{T}$ & This study \\
\hline CMKK-1 & RMKK-1 complemented with CoMEKKI & This study \\
\hline CMKK-2 & RMKK-1 complemented with CoMEKK1 & This study \\
\hline cmkl $\Delta$ & CMK1 deletion mutant of 104-T & Takano et al. 2000 \\
\hline WME4 & 104-T transformed with hyperactive CoMEKK1-4 & This study \\
\hline mME4 & RMKK-1 transformed with hyperactive CoMEKK1-4 & This study \\
\hline cME4 & cmkl $\Delta$ transformed with hyperactive CoMEKK1-4 & This study \\
\hline CG1 & $c m k 1 \Delta$ transformed with $C M K 1$-green fluorescent protein $(G F P)$ fusion gene & This study \\
\hline CG2 & $c m k 1 \Delta$ transformed with $C M K 1-G F P$ fusion gene & This study \\
\hline CG4 & cmk1 $\Delta$ transformed with $C M K 1-G F P$ fusion gene & This study \\
\hline mCG1 & RMKK-1 transformed with $C M K 1-G F P$ fusion gene & This study \\
\hline ACG1 & CG1 transformed with hyperactive CoMEKK1-4 & This study \\
\hline KENH-2 & $\begin{array}{l}\text { comekk1 mutant of 104-T obtained by Agrobacterium tumefaciens-mediated transformation mutant } \\
\text { screening }\end{array}$ & This study \\
\hline
\end{tabular}




\section{Plasmid constructs.}

To create the CoMEKK1 gene-replacement revs-MEKK1AH3 vector, the $\mathrm{pCB}-\mathrm{CoMEKK} 1$ plasmid was constructed by introducing an approximately 4.3-kb HindIII genomic fragment containing CoMEKK1 from a cosmid clone (MEKKcosA10, which contained CoMEKK1) into the HindIII site of pCB1004 (Carroll et al. 1994). An approximately 2.7-kb EZ::TN <KAN$2>$ fragment (the AH3 fragment, in which the kanamycin resistance gene was replaced by the ampicillin resistance gene and the hygromycin resistance gene) was then inserted into pCB-CoMEKK1H. A pCB-MEKK1AH3 plasmid containing the AH3 fragment, 483 bp from the start codon, was selected. Finally, BamHI and ApaI fragments containing the AH3 fragment with CoMEKK1 from the pCB-MEKK1AH3 plasmid were cloned into the $A$. tumefaciens binary vector pBIG4MRSrev, which contained a sulfonylurea resistance gene and was used as the gene replacement plasmid.

To perform a complementation assay of the comekkl mutant, we first constructed the CoMEKK1 compliment vector revs-CoMEKK1. The full region of CoMEKK1 was amplified by PCR with primers CoMEKK1P-S (5'-CGGGATCCGCGG GACAAGGACAACAC-3') and CoMEKK1T-AS (5'-CGTCT AGACGCTTGGTGCGAGAAAAC-3') from the cosmid clone MEKKcosA10. The CoMEKK1P-S primer contained a terminal BamHI site, whereas the CoMEKK1T-AS primer contained a terminal $X b a I$ site. The amplified product was digested with $B a m \mathrm{HI}$ and $\mathrm{XbaI}$, introduced into the Bam HI and $\mathrm{XbaI}$ sites of pBluescriptII SK+, and named pBS-CoMEKK1. Finally, the $B a m \mathrm{HI}$ and $\mathrm{XbaI}$ fragments containing CoMEKKI from the pBS-CoMEKK1 plasmid were cloned into pBIG4MRSrev. To construct the constitutively active Comekk1p expression vector, we constructed pBS-CoMEKK1-4 from pBS-CoMEKK1 using the primer pair Mekk1-4S1 (5'-TCGATTTTGGTATTTCCAA GAAGCT-3') and Mekk1-4AS1 (5'-AATCTTGATGGTGCC CTTG-3') and a KOD-Plus-Mutagenesis Kit (Toyobo, Osaka, Japan) according to the manufacturer's instructions. Finally, the Bam HI and XbaI fragments containing CoMEKK1-4 from the pBS-CoMEKK1-4 plasmid were cloned into pBIG4MRSrev.

To construct the CMK1-GFP fusion plasmid, we first constructed the pBI-BglyGFP plasmid in which the hygromycin resistance gene was replaced with the bialaphos resistance gene from the pBI-HglyGFP plasmid (Sakaguchi et al. 2008). The CMK1 gene containing the full open reading frame of $C M K 1$ with $1 \mathrm{~kb}$ of the $5^{\prime}$ upstream region was amplified by PCR using the primers CM1BMS (5'-CGGGATCCTCGAAG TCTATGGCGTCA-3') and CM1HAS (5'-GACCAAGCTTCC GCATAATCTCCTGGTAGA-3') (Takano et al. 2000). The amplified product was digested with BamHI and HindIII and introduced into pBI-BglyGFP. The resultant clone was designated as $\mathrm{pBI}-\mathrm{CMGFPB}$.

\section{Pathogenicity tests.}

The inoculation assay on cucumber cotyledons (Cucumis sativus L. "Suyo") was performed as described by Tsuji and associates (1997). The conidia of Colletotrichum orbiculare were obtained from 7-day-old cultures and drops $(10 \mu \mathrm{l})$ of conidial suspension $\left(5 \times 10^{5} \mathrm{conidia} / \mathrm{ml}\right)$ were spotted on the surfaces of cucumber cotyledons. To assess invasive growth ability, mycelial blocks incubated on PDA were placed on wound sites prepared by scratching the surfaces of the cotyledons with a sterile toothpick. After inoculation, the cotyledons were incubated at $24^{\circ} \mathrm{C}$ with $16 \mathrm{~h}$ of light per day for 6 days.

\section{Microscopy.}

For appressorium formation assays in vitro, the conidia were harvested from PDA cultures aged 7 days or older and suspended in distilled water or $0.1 \%$ yeast extract solution. The suspension, adjusted to $1 \times 10^{5}$ conidia/ml, was poured onto a multitest glass slide (Glass 8-well multitest slide; ICN Biomedicals, Aurora, OH, U.S.A.) and incubated at $24^{\circ} \mathrm{C}$ in the dark. Germlings were observed with a Nikon ECLIPSE E600 microscope with differential-interference contrast optics. GFP fluorescence was observed on a fluorescent microscope with the GFP(R)-BP filter (460- to 500-nm excitation wavelength, 505-nm wavelength dichroic mirror, and 510- to 560-nm wavelength barrier filter). For staining the nuclei, the conidia were incubated in Hoechst $33342(5 \mathrm{mg} / \mathrm{ml})$ for $5 \mathrm{~min}$. These conidia were washed with distilled water and observed under a fluorescent microscope with a UV-1A filter (excitation wavelength of $365 / 10 \mathrm{~nm}$; dichroic mirror wavelength of $400 \mathrm{~nm}$, and barrier filter wavelength of $400 \mathrm{~nm}$ ).

\section{ACKNOWLEDGMENTS}

This work was supported by Grants-in-Aid for Scientific Research from the Ministry of Education, Culture, Sports, Science \& Technology (nos. 19380029 and 21380031)

\section{LITERATURE CITED}

Andrews, D. L., Egan, J. D., Mayorga, M. E., and Gold, S. E. 2000. The Ustilago maydis ubc4 and ubc5 genes encode members of a MAP kinase cascade required for filamentous growth. Mol. Plant-Microbe Interact. 13:781-786.

Asakura, M., Ninomiya, S., Sugimoto, M., Oku, M., Yamashita, S., Okuno, T., Sakai, Y., and Takano, Y. 2009. Atg26-mediated pexophagy is required for host invasion by the pla1291-nt pathogenic fungus Colletotrichum orbiculare. Plant Cell 21:1291-1304.

Banuett, F., and Herskowitz, I. 1994. Identification of fuz7, a Ustilago maydis MEK/MAPKK homologue required for a locus-dependent and -independent steps in the fungal life cycle. Genes Dev. 8:1367-1378.

Blackwell, E., Halatek, I. M., Kim, H. J., Ellicott, A. T., Obukhov, A. A., and Stone, D. E. 2003. Effect of the pheromone-responsive G(alpha) and phosphatase proteins of Saccharomyces cerevisiae on the subcellular localization of the Fus3 mitogen-activated protein kinase. Mol. Cell Biol. 23:1135-1150.

Bruno, K. S., Tenjo, F., Li, L., Hamer, J. E., and Xu, J. R. 2004. Cellular localization and role of kinase activity of PMK1 in Magnaporthe grisea. Eukaryot. Cell 3:1525-1532.

Carroll, A. M., Sweigard, J. A., and Valent, B. 1994. Improved vectors for selecting resistance to hygromycin. Fungal Genet. Newsl. 41:22-23.

Chen, R. E., and Thorner, J. 2007. Function and regulation in MAPK signaling pathways: Lessons learned from the yeast Saccharomyces cerevisiae. Biochim. Biophys. Acta 1773:1311-1340.

Fujihara, N., Sakaguchi, A., Tanaka, S. Fujii, S., Tsuji, G., Shiraishi, T., O'Connell R., and Kubo, Y. Peroxisome biogenesis factor PEX13 is required for appressorium-mediated plant infection by the anthracnose fungus, Colletotrichum orbiculare. Mol. Plant-Microbe Interact. 4:436-445.

Grimshaw, S. J., Mott, H. R., Stott, K. M., Nielsen, P. R., Evetts, K. A., Hopkins, L. J., Nietlispach, D., and Owen, D. 2004. Structure of the sterile alpha motif (SAM) domain of the Saccharomyces cerevisiae mitogen-activated protein kinase pathway-modulating protein STE50 and analysis of its interaction with the STE11 SAM. J. Biol. Chem. 279:2192-2201.

Kimura, A., Takano, Y., Furusawa, I., and Okuno, T. 2001. Peroxisomal metabolic function is required for appressorium-mediated plant infection by Colletotrichum lagenarium. Plant Cell 13:1945-1957.

Kojima, K., Kikuchi, T., Takano, Y., Oshiro, E., and Okuno, T. 2002. The mitogen-activated protein kinase gene $M A F 1$ is essential for the early differentiation phase of appressorium formation in Colletotrichum lagenarium. Mol. Plant- Microbe Interact. 15:1268-1276.

Kojima, K., Takano, Y., Yoshimi, A., Tanaka, C., Kikuchi, T., and Okuno, T. 2004. Fungicide activity through activation of a fungal signalling pathway. Mol. Microbiol. 53:1785-1796.

Kubo, Y., and Furusawa, I. 1991. Melanin biosynthesis: Prerequisite for successful invasion of the plant host by appressoria of Colletotrichum and Pyricularia. Pages 205-217 in: The Fungal Spore and Disease Initiation in Plants and Animals. G. T. Cole and H. C. Hoch, (eds. Plenum Publishing Corporation, New York.

Kubo, Y., Takano, Y., Endo, N., Yasuda, N., Tajima, S., and Furusawa, I. 1996. Cloning and structural analysis of the melanin biosynthesis gene SCD1 encoding scytalone dehydratase in Colletotrichum lagenarium. Appl. Environ. Microbiol. 62:4340-4344. 
Li, L., Xue, C. Y., Bruno, K., Nishimura, M., and Xu, J. R. 2004. Two PAK kinase genes, CHM1 and MST20, have distinct functions in Magnaporthe grisea. Mol. Plant-Microbe Interact. 17:547-556.

Liu, Y. G., Mitsukawa, N., Oosumi, T., and Whittier, R. F. 1995. Efficient isolation and mapping of Arabidopsis thaliana T-DNA insert junctions by thermal asymmetric interlaced PCR. Plant J. 8:457-463.

Mayorga, M. E., and Gold, S. E. 1998. Characterization and molecular genetic complementation of mutants affecting dimorphism in the fungus Ustilago maydis. Fungal Genet. Biol. 24:364-376.

Mayorga, M. E., and Gold, S. E. 1999. A MAP kinase encoded by the ubc3 gene of Ustilago maydis is required for filamentous growth and full virulence. Mol. Microbiol. 34:485-497.

Müller, P., Aichinger, C., Feldbrugge, M., and Kahmann R. 1999. The MAP kinase kpp2 regulates mating and pathogenic development in Ustilago maydis. Mol. Microbiol. 34:1007-1017.

Müller, P., Weinzierl, G., Brachmann, A., Feldbrügge, M., and Kahmann, R. 2003. Mating and pathogenic development of the Smut fungus Ustilago maydis are regulated by one mitogen-activated protein kinase cascade. Eukaryot. Cell 2:1187-1189.

Perpetua, N. S., Kubo, Y., Yasuda, N., Takano, Y., and Furusawa, I. 1996. Cloning and characterization of a melanin biosynthetic THR1 reductase gene essential for appressorial penetration of Colletotrichum lagenarium. Mol. Plant-Microbe Interact. 9:323-329.

Posas, F., and Saito, H. 1997. Osmotic activation of the HOG MAPK pathway via Ste11p MAPKKK: Scaffold role of Pbs2p MAPKK. Science 276:1702-1705.

Reiser, V., Ruis, H., and Ammerer, G. 1999. Kinase activity-dependent nuclear export opposes stress-induced nuclear accumulation and retention of Hog1 mitogen-activated protein kinase in the budding yeast Saccharomyces cerevisiae. Mol. Biol. Cell. 10:1147-1161.

Sakaguchi, A., Miyaji, T., Tsuji, G., and Kubo, Y. 2008. Kelch repeat protein Clakel2p and calcium signaling control appressorium development in Colletotrichum lagenarium. Eukaryot. Cell 7:102-111.

Sakaguchi, A., Miyaji, T., Tsuji, G., and Kubo, Y. 2010. A Kelch repeat protein Cokel1p associates with microtubules and is involved in appressorium development in Colletotrichum orbiculare. Mol. Plant-Microbe Interact. 23:103-111.

Sambrook, J., Fritsch, E. F., and Maniatis, T. 1989. Molecular Cloning: A Laboratory Manual. Cold Spring Harbor Laboratory Press, Cold Spring Harbor, NY, U.S.A.

Stevenson, B. J., Rhodes, N., Errede, B., and Sprague, G. F., Jr. 1992 Constitutive mutants of the protein kinase STE11 activate the yeast pheromone response pathway in the absence of the $\mathrm{G}$ protein. Gene Dev. 6:1293-1304.

Stork, P. J. S., and Schmitt, J. M. 2002. Crosstalk between cAMP and MAP kinase signaling in the regulation of cell proliferation. Trends Cell Biol. 12:258-266.

Takano, Y., Kubo, Y., Shimizu, K., Mise, K., Okuno, T., and Furusawa, I. 1995. Structural analysis of PKS1, a polyketide synthase gene involved in melanin biosynthesis in Colletotrichum lagenarium. Mol. Gen. Genet. 249:162-167.

Takano, Y., Kubo, Y., Kawamura, C., Tsuge, T., and Furusawa, I. 1997. The Alternaria alternata melanin biosynthesis gene restores appressorial melanization and penetration of cellulose membranes in the melanin deficient albino mutant of Colletotrichum lagenarium. Fungal Genet. Biol. 21:131-140.

Takano, Y., Kikuchi, T., Kubo, Y., Hamer, J. E., Mise, K., and Furusawa,
I. 2000. The Colletotrichum lagenarium MAP kinase gene CMK1 regulates diverse aspects of fungal pathogenesis. Mol. Plant-Microbe Interact. 13:374-383

Takano, Y., Komeda, K., Kojima, K., and Okuno, T. 2001. Proper regulation of cyclic AMP-dependent protein kinase is required for growth, conidiation, and appressorium function in the anthracnose fungus $\mathrm{Col}$ letotrichum lagenarium. Mol Plant-Microbe Interact. 14:1149-57.

Tanaka, S., Yamada, K., Yabumoto, K., Fujii, S., Huser, A., Tsuji, G., Koga, H., Dohi, K., Mori, M., Shiraishi, T., O’Connell, R., and Kubo, Y. 2007. Saccharomyces cerevisiae SSD1 orthologues are essential for host infection by the ascomycete plant pathogens Colletotrichum lagenarium and Magnaporthe grisea. Mol. Microbiol. 64:1332-1349.

Tanaka, S., Ishihama, N., Yoshioka, H., Huser, A., O'Connell, R., Tsuji, G., Tsuge, S., and Kubo, Y. 2009. The Colletotrichum orbiculare ssd1 mutant enhances Nicotiana benthamiana basal resistance by activating a mitogen-activated protein kinase pathway. Plant Cell 21:2517-2526.

Tsuji, G., Takeda, T., Furusawa, I., Horino, O., and Kubo, Y. 1997. Carpropamid, an anti-rice blast fungicide, inhibits scytalone dehydratase activity and appressorial penetration in Colletotrichum lagenarium. Pestic. Biochem. Physiol. 57:211-219.

Tsuji, G., Kenmochi, Y., Takano, Y., Sweigard, J., Farrall, L., Furusawa, I. Horino, O., and Kubo, Y. 2000. Novel fungal transcriptional activators, Cmr1p of Colletotrichum lagenarium and Pig1p of Magnaporthe grisea, contain Cys2His2 zinc finger and Zn(II)2Cys6 binuclear cluster DNA binding motifs, and regulate transcription of melanin biosynthesis genes in a developmentally specific manner. Mol. Microbiol. 38:940954.

Tsuji, G., Fujii, S., Fujihara, N., Hirose, C., Tsuge, S., Shiraishi, T., and Kubo, Y. 2003a. Agrobacterium tumefaciens-mediated transformation for random insertional mutagenesis in Colletotrichum lagenarium. J. Gen. Plant Pathol. 69:230-239.

Tsuji, G., Fujii, S. Tsuge, S., Shiraishi, T., and Kubo, Y. 2003b. The Colletotrichum lagenarium Ste12-like gene CST1 is essential for appressorium penetration. Mol. Plant-Microbe Interact. 16:315-325.

van Drogen, F., O'Rourke, S. M., Stucke, V. M., Jaquenoud, M., Neiman, A. M., and Peter, M. 2000. Phosphorylation of the MEKK Ste11p by the PAK-like kinase Ste20p is required for MAP kinase signaling in vivo. Curr. Biol. 10:630-639.

Xu, J. R. 2000. Map kinases in fungal pathogens. Fungal Genet. Biol 31:137-152.

Xu, J. R., Zhao, X., and Dean, R. A. 2007. From genes to genomes: A new paradigm for studying fungal pathogenesis in Magnaporthe oryzae. Adv. Genet. 57:175-218.

Yamauchi, J., Takayanagi, N., Komeda, K., Takano, Y., and Okuno, T. 2004. cAMP-pKA signaling regulates multiple steps of fungal infection cooperatively with Cmk1 MAP kinase in Colletotrichum lagenarium. Mol. Plant-Microbe. Interact. 17:1355-1365.

Zhao, X., Kim, Y., Park, G., and Xu, J. R. 2005. A mitogen-activated protein kinase cascade regulating infection-related morphogenesis in Magnaporthe grisea. Plant Cell 17:1317-1329.

Zhao, X., Mehrabi, R., and Xu, J. R. 2007. Mitogen-activated protein kinase pathways and fungal pathogenesis. Eukaryot. Cell 6:1701-1714.

\section{AUTHOR-RECOMMENDED INTERNET RESOURCE}

TreeView software: taxonomy.zoology.gla.ac.uk/rod/treeview.html 